IFT-UAM/CSIC-99-28

hep-th/9907205

\title{
Ultraviolet and Infrared Freedom from String Amplitudes
}

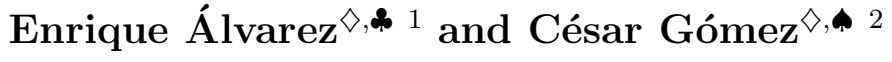 \\ $\diamond$ Theory Division, CERN,1211 Geneva 23, Switzerland \\ and \\ Instituto de Física Teórica, C-XVI, Universidad Autónoma de Madrid \\ E-28049-Madrid, Spain[ \\ - Departamento de Física Teórica, C-XI, Universidad Autónoma de Madrid \\ E-28049-Madrid, Spain \\ - I.M.A.F.F., C.S.I.C., Calle de Serrano 113 \\ E-28006-Madrid, Spain
}

\begin{abstract}
The infinite tension limit of string amplitudes is examined with some care, identifying the part responsible of diamagnetic behaviour as well as a peculiar paramagnetic tachyon magnifying responsible of aysmptotic freedom. The way string theory represents abelian gauge theories is connected with the non-planar reggeon/pomeron amplitude and a nontrivial beta function is found in the low energy limit of a single D-brane.
\end{abstract}

\footnotetext{
${ }^{1}$ E-mail: enrique.alvarez@uam.es

${ }^{2}$ E-mail: iffgomez@roca.csic.es

${ }^{3}$ Unidad de Investigación Asociada al Centro de Física Miguel Catalán (C.S.I.C.)
} 


\section{Introduction}

One of the most important results in quantum field theory was the discovery [11] of asymptotic freedom for non abelian gauge groups. String theory, among other things, can be used as a new regulator of quantum field theories with the ultraviolet cutoff given by the string tension $\alpha^{\prime}$. Appropiated field theory limits, $\alpha^{\prime}=0$, can be defined for string amplitudes and there is now an impressive body of knowledge (cf. for example, [12], [13]. [8], [5], and references therein) on the recovering, in this limit, of the perturbative series of non abelian gauge theories and in particular the correct asymptotically free beta function.

At the level of open string amplitudes the difference between dealing with abelian or non abelian gauge groups reduces simply to the type of Chan-Paton factors used to decorate the end points of the open string. These group theoretical elements appear in string theory as multiplicative factors of the skeleton string amplitude that are independent of the gauge group. Thus from the string point of view the only difference between abelian and non abelian gauge theories stems basically from the existence of a no planar contribution to the two point amplitude in the abelian case that vanishes for non abelian Chan-Paton factors. Therefore and using string theory as the driving principle we notice that the appearance of infrared free theories is intimately related to the existence of the old fashion reggeonpomeron amplitude on the basis of which we build up the the two point non planar string diagram.

Our first aim in this paper is to perform a careful analysis, in the infinite tension limit, of some open string two point functions and to unravel the specific way string theory produces infrared free beta functions. From this analysis we shall get some interesting physical results.

First of all we observe that string theory produces a decomposition of the beta function into two pieces, infrared free and asymptotically free respectively, corresponding exactly to the diamagnetic and paramagnetic contributions to the pure Yang Mills beta func- 
tion ([14). When we take into account the non planar two point function -specific to abelian Chan-Paton factors- we cancel the paramagnetic asymptotically free part ending up with a non trivial infrared free beta function corresponding to scalar quantum electrodynamics (QED) with $(d-2)$ charged scalar fields, in $d$ space-time dimensions. We then observe that the way strings, manage to produce infrared free behavior is through the non planar (reggeon/pomeron vertex) contribution which precisely cancels the paramagnetic (spin-dependent) piece of the pure Yang Mills beta function, while keeping untouched the diamagnetic part which shows up eventually as associated with a set of unexpected charged scalar fields.

In modern terminology Chan-Paton factors become equivalent to D-branes and the difference between non abelian and abelian equivalent to that between a family of parallel $\mathrm{D}$ (irichlet)-branes and one isolated D-brane.

Using D-3 branes we can repeat our analysis for the beta function in the infinite tension limit, but this time for the open string amplitude with world sheet boundaries located on the D-3 brane world volume. For the non abelian case we get -at one loop- the expected result, namely the Yang Mills beta function for a theory with $(D-4)$ charged scalars in the adjoint representation (where $D$ is the external spacetime dimension). It is interesting to notice that this beta function precisely vanishes for $D$ the bosonic string critical dimension 26. Moreover the so derived beta function agrees with the expected spectrum on a set of parallel D-3 branes in the bosonic string, namely Yang Mills gluons and adjoint charged scalars, one for each transverse direction (the Goldstone bosons of the spontaneously broken translation invariance).

For the case of one D-brane and once we take into account the non planar contribution to the two point function, we get a beta function for scalar QED with $(D-2)$ charged scalars, a matter content with an unclear geometrical interpretation. In any case we notice that what will make the world volume dynamics on one D-brane infrared free is intimately related to the non vanishing amplitude for spontaneous emission processes of the type 
depicted in figure 1.
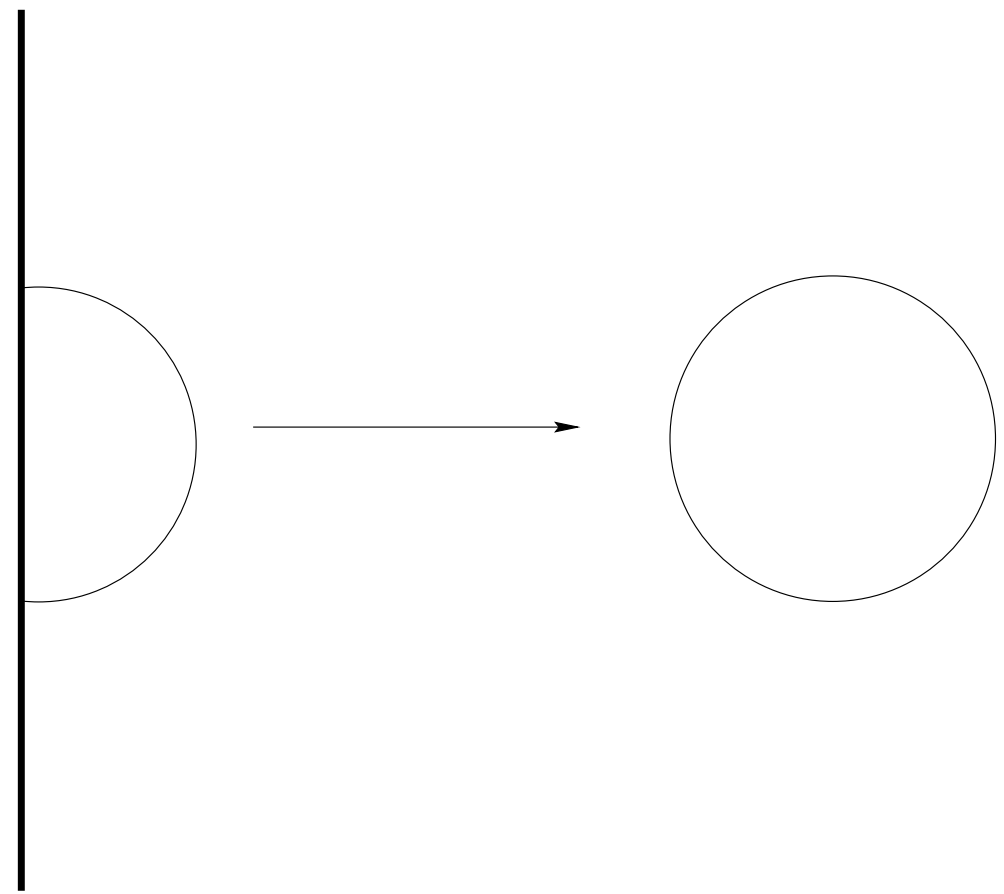

Figure 1: Open/closed string transition from D-branes to the bulk

If by some stability argument we can freeze this emission we will end up with an asymptotically free dynamics on the world volume of the D-brane.

\section{Field Theory Limit of String Amplitudes}

String techniques have been successfully used in the last decade to reproduce field theory amplitudes in a way leading to further insights in the computation of field theory Feynman diagrams. (cf. [5]).

In order to be specific, let us start by reviewing the recipe of the field theory limit in the simplest setting aiming to deriving the $S U(N)$ gluon propagator from the open bosonic string (cf. 8 ). 
This amplitude is given by

$$
C_{a b}\left(k_{1}, k_{2}\right) \equiv \operatorname{Tr}<\epsilon_{1}^{\mu} V_{a \mu}\left(k_{1}\right) \epsilon_{2}^{\nu} V_{b \nu}\left(k_{2}\right)>
$$

where the gauge vector vertex operator is given by

$$
V_{a}^{\mu}(k) \equiv g_{Y M} \sqrt{2 \alpha^{\prime}} T_{a} \frac{d x^{\mu}}{d \lambda} e^{i k \cdot X(\lambda)}
$$

the matrices $T_{a}$ being the generators of the gauge group $S U(N)$. The parameter $\lambda$ indicates the insertion point on the boundary of the world surface of the string, and has to be integrated over. The Yang-Mills coupling constant is related to the string coupling, $g_{s}$, through the D-brane relationship $g_{Y M}=2\left(2 \alpha^{\prime}\right)^{\frac{d-4}{4}} g_{s} \equiv 2^{\frac{d}{4}} l_{s}^{\frac{d-4}{2}} g_{s}$.The simplest one loop contribution is the annulus (which is topologically a cylinder), which we shall parametrize, following [10] by the annular region shown in the figure, with the two arcs identified in the sense shown by the arrows. (This is gotten by defining the convenient variables $\omega \equiv$ $\lambda_{1} \lambda_{2} \in(0,1)$ and $\left.\rho \equiv \lambda_{1} \in(\omega, 1)\right)$.

The modulus of the cylinder is $\tau \equiv-\frac{1}{2} \log \omega \in(0, \infty)$ (whereas $\omega \in(0,1)$ ). Instead of the insertion point on the boundary $\rho \in(\omega, 1)$, we will use the convenient variable $\nu \equiv-\frac{1}{2} \log \rho$.

The diagrams can be classified as planar and non-planar, depending on whether the two vertex operators are inserted on the same boundary (planar) or in different boundaries (non planar). We normalize the generators by $\operatorname{tr} T_{a} T_{b} \equiv C_{2}^{f u n d}(G) \delta_{a b}=\frac{1}{2} \delta_{a b}$.

The infinite tension limit $\alpha^{\prime} \rightarrow 0$ is expected to be dominated by configurations in which $\tau \rightarrow \infty$. This corresponds to cylinders with divergent section (that is, infinite time from the open string point of view, and only the massless open string states can survive in this limit).This is then the natural infrared limit from the open string point of view.

Owing to conformal invariance, the situation depicted is equivalent to a cylinder with constant section, and length $l=\frac{1}{\tau}$. This would be the most natural way to look at the cylinder from the closed string channel point of view. The limit we are taking is then the limit in which the length of this cylinder goes to zero. 

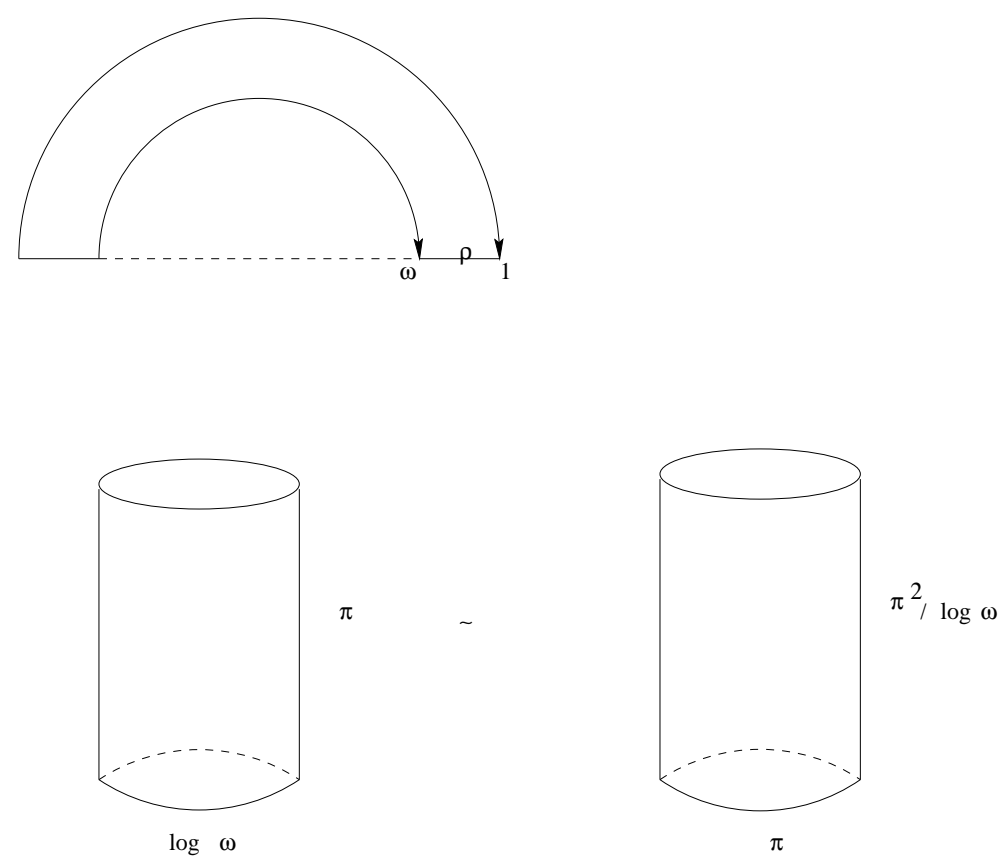

Figure 2: The annular region of integration for the one-loop diagram

The planar amplitude (which gives the full contribution of the simple group $G=S U(N)$ under consideretion here) is given by $(\llbracket 10)$ f:

$$
\begin{aligned}
& C_{a b}\left(k_{1}, k_{2}\right)=N \frac{g^{2} \mu^{4-d}}{(4 \pi)^{d / 2}} \frac{1}{2} \delta_{a b}\left(2 \alpha^{\prime}\right)^{1-d / 2} \\
& \int_{0}^{\infty} d \tau e^{\frac{(26-d) \tau}{12}} \eta(i \tau / \pi)^{-(d-2)} \int_{0}^{\tau} d \nu \tau^{-d / 2} \\
& {\left[-\left(\epsilon_{1} \cdot \epsilon_{2}\right) \partial_{\nu_{1}} \partial_{\nu_{2}} G\left(\nu_{1}, \nu_{2}\right)+\alpha^{\prime}\left(\epsilon_{1} \cdot p_{2}\right)\left(\epsilon_{2} \cdot p_{1}\right) \partial_{\nu_{1}} G\left(\nu_{1}, \nu_{2}\right) \partial_{\nu_{2}} G\left(\nu_{1}, \nu_{2}\right)\right]} \\
& e^{2 \alpha^{\prime} p_{1} \cdot p_{2} G\left(\nu_{1}, \nu_{2}\right)}
\end{aligned}
$$

\footnotetext{
${ }^{2}$ We follow the explicit normalization of Di Vecchia et al. in [8]; including in particular their prescription for an off-shell continuation, namely, to gauge-fix the projective transformations on the Koba-Nielsen variables and to continue the momenta to $k_{i}^{2}=M^{2}$, while keeping transversality, $\epsilon_{i} k_{i}=0 \forall i$. This prescription is different from the one advocated in [5], but it is much simpler for our purposes, and it has not led to any known inconsistencies.
} 
$G\left(\nu_{1}, \nu_{2}\right)$ is the standard 1 Neumann Green function (cf. [10]),

$$
G(\nu \mid \tau) \equiv \log -2 \pi i \frac{\theta_{1}\left(i \frac{\nu}{\tau} \mid i \frac{\tau}{\pi}\right)}{\theta_{1}^{\prime}\left(0 \mid i \frac{\tau}{\pi}\right)}-\frac{\nu^{2}}{\tau}
$$

There are many points of interest in this formula. The traces over the Chan-Paton on the two boundaries give $\operatorname{tr} 1=N$ for the boundary free of external states and $\operatorname{tr} T_{a} T_{b}=\frac{1}{2} \delta_{a b}$ for the boundary where the vertex operators lie.

The amplitude has been boldly written in arbitrary dimension $d$, in spite of the fact that we know that the string theory is only unitary if $d=26$. We will eventually be intested in $d=4$, and we are expressing the coupling constant in terms of the dimensionless fourdimensional one, so that we have included a term $\mu^{4-d}$.

A further point is that, on shell, by momentum conservation, $p_{1}=-p_{2}$, so that $p_{1}^{2}=$ $p_{2}^{2}=p_{1} \cdot p_{2}=p_{1} \epsilon_{1}=p_{2} \epsilon_{2}=0$, and the only surviving term is the one involving a second derivative of the Green's function (which gives zero upon integration).

The standard procedure (see [0] [8]) is to integrate by parts precisely this term, and stay with the rest, making analytic continuation in the Mandelstam variable

$$
s \equiv p_{1} \cdot p_{2}
$$

In order to take the infinite tension limit of the amplitudes, we shall take simultaneously $\alpha^{\prime}, \tau^{-1} \rightarrow \infty$, un such a way that the combination $\bar{\tau} \equiv \alpha^{\prime} \tau$ stays finite. Actually, this same variable $\bar{\tau}$ will play the rôle of Schwinger's proper time in the field theory limit. Let us stress that due to the conformal mapping depicted in Figure 2, the $\tau^{-1}$ is the world sheet length of the cylinder, so that the limit $\tau \rightarrow \infty$ corresponds to zero length where the dominant contribution corresponds to the lightest open string states.

\footnotetext{
${ }^{3}$ This Green function is $2 \pi$ times the one used in [6]

$$
G_{B M}(z \mid \tau) \equiv \log \left|\frac{\theta_{1}(z \mid \tau)}{\theta_{1}^{\prime}(0 \mid \tau)}\right|-\pi \frac{(\operatorname{Im} z)^{2}}{\operatorname{Im} \tau}
$$

with the important difference that it does not have modulus in the logarithm. Note in particular that $G_{B M}(z+\tau \mid \tau)=G_{B M}(z \mid \tau)$, whereas $G(\nu+\tau \mid \tau)=G(\nu \mid \tau)+i \pi$
} 
In this limit, the contribution of the partition function (conveyed by Dedekind's function) is given by:

$$
1+(d-2) e^{-2 \tau}
$$

corresponding to the open tachyon and gluon contribution to the loop. Once we multiply (可) by the measure factor $e^{2 \tau}$ we get the standard tachyon divergence as well as a finite contribution for the gluonic piece in (7). The terms involving the Green's function can be represented by:

$$
R(s)=\frac{1}{s} \int_{0}^{\infty} \frac{d \tau}{\tau}\left[e^{2 \tau}+(d-2)\right] \int_{0}^{\tau} d \nu \tau^{1-d / 2} e^{2 \alpha^{\prime} s G(\nu)}\left(\partial_{\nu} G(\nu)\right)^{2}
$$

\subsection{The Tachyon magnification}

The large $\tau$ expansion of the Green function is:

$$
G(\nu)=-\frac{\nu^{2}}{\tau}+\nu+\log \left(1-e^{-2 \nu}\right)+e^{-2 \tau}\left[3+\frac{e^{-3 \nu}-e^{3 \nu}}{e^{\nu}-e^{-\nu}}\right]
$$

which can as well be written as:

$$
G(\nu)=-\frac{\nu^{2}}{\tau}+\nu-\sum_{n=1}^{\infty} \frac{e^{-2 n \nu}}{n}+e^{-2 \tau}\left[3-\sum_{n=0}^{\infty} e^{2(1-n) \nu}+\sum_{n=0}^{\infty} e^{-2(n+2) \nu}\right]
$$

Let us now consider the region $\nu=\tau \rightarrow \infty$ as defining a new integration variable $\hat{\nu} \equiv \nu / \tau$. The relevant contribution to the integrand of (8) is given by:

$\left[e^{2 \tau}+(D-2)\right](\partial G)^{2}=(D-2)\left(1-2 \hat{\nu}^{2}\right)+4 e^{-4 \hat{\nu} \tau}+4(1-2 \hat{\nu}) e^{-2 \hat{\nu} \tau}-8+4 e^{(4 \hat{\nu}-2) \tau}-4(1-2 \hat{\nu}) e^{2 \hat{\nu} \tau}$

The correct procedure in this limit, according to the above rules is to neglect the two last terms of the above expansion, and then, perform the integration over $d \hat{\nu}$ of the remaining integrand. Let us insist that we have to neglect the term $4 e^{(4 \hat{\nu}-2) \tau}$, in spite of the fact that it enjoys a finite limit when $\tau \rightarrow \infty$ as long as $\hat{\nu} \leq 1 / 2$. This means that it is necessary to neglect as tachyon artifacts all terms that diverge when $\tau \rightarrow \infty$ for some value of $\hat{\nu}$. 
The simplest remaining term, proportional to $(d-2)$, comes from the zero mode part of the Green function, and is given by:

$$
e^{2 \alpha^{\prime} s\left(\hat{\nu}-\hat{\nu}^{2}\right) \tau}(d-2)(1-2 \hat{\nu})^{2}
$$

The remaining contribution, which can be interpreted as due to some sort of tachyon magnification (because there is a cancellation of the divergent contribution of the tachyon with an exponentially suppressed contribution coming from oscillatory modes in the Green function), just gives:

$$
e^{2 \alpha^{\prime} s\left(\hat{\nu}-\hat{\nu}^{2}\right) \tau}[-8]
$$

It should be stressed that by working in the combined limit $\alpha^{\prime}, \tau^{-1}, \nu^{-1} \rightarrow \infty$, with the blow-up variables $\tilde{\tau} \equiv \alpha^{\prime} \tau$ and $\hat{\nu} \equiv \nu / t$, we have avoided the singular region in moduli space corresponding to coincident vertex operators (cf. Figure 3), which from the field theory point of view corresponds to the two last diagrams of Figure 4, which do not contribute to the propagator.

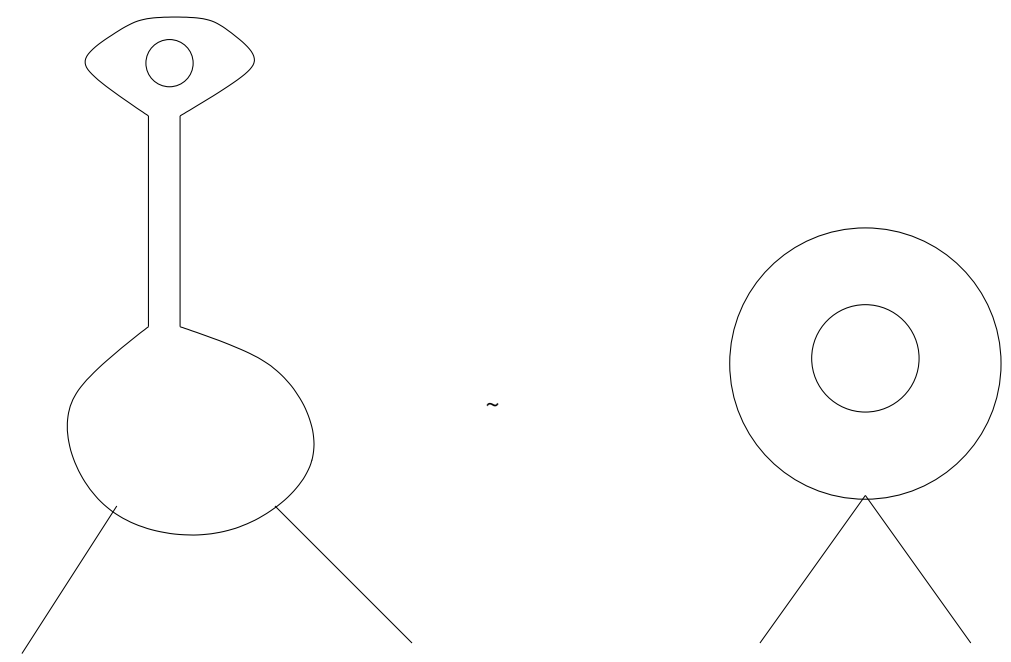

Figure 3: Two conformally equivalent ways of depicting coincident vertex operators

All this yields

$$
R(s)=-\Gamma(2-d / 2)\left(-2 \alpha^{\prime} s\right)^{d / 2-2} \frac{7 d-6}{d-1} B(d / 2-1, d / 2-1)
$$


(where $B(z, w)$ is Euler's beta function). It is useful for later purposes to realize that

$$
\frac{7 d-6}{d-1}=8-\frac{d-2}{d-1} .
$$
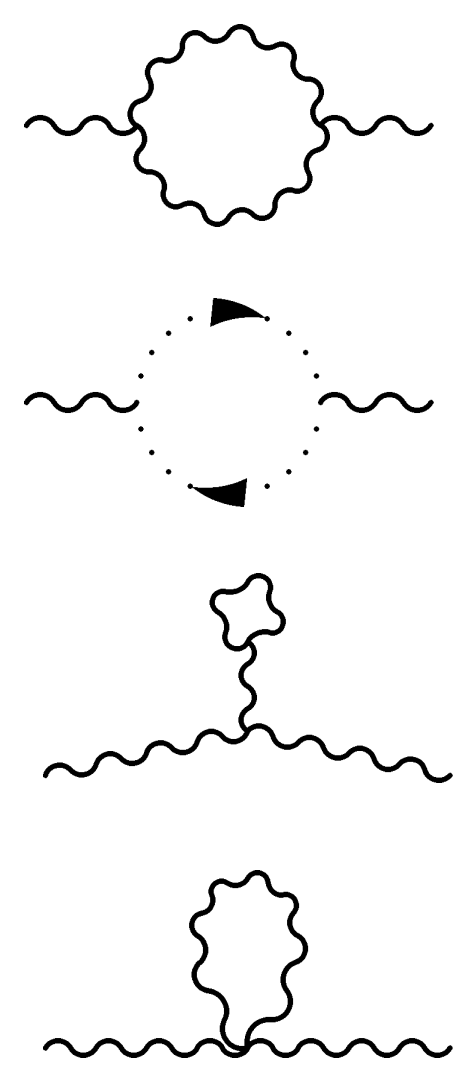

Figure 4: Diagrams for the Gluon Propagator. The wavy line represents gluons; the discontinuous one, ghosts.

The final result of the (divergent part of the )two-gluon amplitude, computed in this way, is:

$$
\begin{aligned}
& C_{a b}(s)=\frac{N}{2} \delta_{a b} \frac{g^{2}}{(4 \pi)^{d / 2}}\left(2 \alpha^{\prime}\right)^{2-d / 2}\left(\epsilon_{1} . \epsilon_{2}\right) s R(s)= \\
& -\frac{N}{2} \delta_{a b} \frac{g^{2}}{(4 \pi)^{2}} \epsilon_{1} \cdot \epsilon_{2} s \Gamma(\epsilon) \frac{22}{3} B(1,1)
\end{aligned}
$$


(where in the second line dimensional regularization around $d=4$ has been used). It is remarkable that this is exactly the background field Feynman gauge result. Indeed, using the Ward identity $Z_{g}=Z_{A}^{-1 / 2}$, one gets

$$
g_{0} \equiv \mu^{\epsilon} \sum_{n=0}^{\infty} \frac{a_{n}(g)}{\epsilon^{n}}=g \mu^{\epsilon}\left(1-N \frac{g^{2}}{32(\pi)^{2}} \frac{11}{3} \frac{1}{\epsilon}\right)+0\left(\frac{1}{\epsilon^{2}}\right)
$$

This leads to the correct gauge theory $\beta$ function, namely:

$$
\beta(g) \equiv \mu \frac{\partial g}{\partial \mu}=-a_{1}+g \frac{\partial}{\partial g} a_{1}=-\frac{11}{3} C_{2}^{a d j}(G) \frac{g^{3}}{16 \pi^{2}}
$$

(where $\left.C_{2}^{a d j}(G=S U(N))=N\right)$.

Indeed, the $\frac{11}{3}=4-\frac{1}{3}$ 团, where the infrared free part comes from the $(d-2) e^{-2 \tau}$ part in the expansion of the Dedekind function, and the whole ultraviolet free part comes from the 1 (that is, the tachyon magnification).

This whole setup is to be contrasted with the corresponding calculation in QFT, where both the contributions of the ghosts and the vector bosons are UV free $(11 / 3=10 / 3+$ $1 / 3)$.

\subsection{The rôle of the off-shell extension}

It has already been pointed out that the amplitude (16) is zero on shell, so that it had to be continued off-shell to get a non-vanishing result. This step is a perfectly banal one in field theory but full of dangers instead in string theory, owing to the fact that only on shell are the vertex operators conformal fields of scale dimension $(1,1)$, which is in turn necessary for consistency.

In the language of the operator formalism ( $[4]$, the on-shell condition on the external states is necessary to prove that physical amplitudes are independent of the local coordinates of the punctures corresponding to the insertions of the vertex operators. When the

\footnotetext{
${ }^{4}$ This decomposition was previously found (in the form $\left.(D-26) / 24=(D-2) / 24-1\right)$ in [12]) when they were looking for the string theory effective action.
} 
amplitude is extended off-shell, changes of the coordinates of the punctures do change the physical amplitudes. In reference ([8]) it has been argued that in the field theory limit defined as above, those changes are equivalent to changes of gauge fixing.

The fact that we recover in this way the background field Feynman gauge (with the associated Ward identity) explains why we were able to extract the beta function from the gluon propagator alone. Although we will have no use for it, it is also true that tree and loop amplitudes are naturally recovered in different gauges, another peculiar property of the background field (cf. the second reference in [1]).

\section{Stringy interpretation of asymptotic freedom}

It would be very interesting to find a field theory interpretation of the natural string decomposition of the beta function in a infrared free part (coming fromn the zero modes) and the asymptotically free piece, visible only thanks to the tachyon magnification. It appears that this decomposition naturally fits a beautiful heuristic argument attributed to 't Hooft by N.K. Nielsen (cf. [14]), where the two pieces are called dia and paramagnetic because of the striking similarity of the gauge theory vacuum with a polarizable medium.

Let us start by expanding the gauge field around a background $\bar{A}_{\mu}$ :

$$
A_{\mu}=\bar{A}_{\mu}+a_{\mu}
$$

so that

$$
F_{\mu \nu}=\bar{F} \mu \nu+\bar{\nabla}_{\mu} a_{\nu}-\bar{\nabla}_{\nu} a_{\mu}+\left[a_{\mu}, a_{\nu}\right]
$$

The Yang-Mills Lagrangian then reads:

$$
\begin{aligned}
& L=-\frac{1}{4} \bar{F}_{\mu \nu} \bar{F}^{\mu \nu}-\frac{1}{2} \bar{F}_{\mu \nu}\left(\bar{\nabla}_{\mu} a_{\nu}-\bar{\nabla}_{\nu} a_{\mu}\right)-\frac{1}{2}\left(\bar{\nabla}_{\mu} a_{\nu}\right)^{2}-\frac{1}{2} \bar{F}^{\mu \nu}\left[a_{\mu}, a_{\nu}\right] \\
& +\frac{1}{2}\left(\bar{\nabla}_{\mu} a_{\mu}\right)^{2}-\frac{1}{2}\left(\bar{\nabla}_{\mu} a_{\nu}-\bar{\nabla}_{\nu} a_{\mu}\right)\left[a_{\mu}, a_{\nu}\right]
\end{aligned}
$$

The terms linear in the quantum fields vanish if the background field is on shell. On the other hand, up to the one loop approximation, only terms quadratic in the quantum fields 
do contribute. Now it is clear that the diamagnetic (i.e., infrared free) part will come from the covariant derivative term; actually, the current in the loop induces, via the well-known Lenz's law, a magnetic moment which tends to cancel the external magnetic field; this leads to the screening of the charge or what is the same, in QFT language, to infrared freedom.

After including the effects of the gauge fixing $\bar{\nabla}_{\mu} a_{\mu}=0$, embodied in the ghost fields, this part of the amplitude (up to multiplicative group theory factors) goes as the vacuum polarization in scalar QED with $d-2$ scalars (the true physical polarizations). This is exactly the contribution we get in string theory coming from the zero modes which corresponds to the $1 / 3$ in the former units.

The paramagnetic term, coming from the spin dependent interaction (the last term in equation (21)) (and where, we insist, all the asymptotic freedom resides) matches correspondingly the tachyon magnification term from the stringy viewpoint that is, the -4 in the same units as before. It is hard to believe that this is only a coincidence; everything points in the direction that there is still much to be learned on the physical meaning of asymptotic freedom from the study of string amplitudes.

\section{Physical effect of D-branes}

We have considered up to now formal string amplitudes in $d=4$ dimensions. In spite of the unavoidable off-shell continuation, in the limit $\tau \rightarrow \infty$ we have encountered no problems with unitarity, nor any other inconsistency.

There is, however another possibility, namely to work with critical strings (i.e., $D=26$ ), and still consider gluonic amplitudes on a D- $p=3$ brane world volume. It will prove useful to work out most formulas for generic $(D, d)$.

Old-fashioned Chan-Paton factors are transformed in a sense, in Dirichlet boundary conditions for open strings, the $U(N)$ gauge theory being thus represented by a stack of 
$N$ parallel D-branes. The standard lore is to argue that the $U(1)$ part just represents the center of mass degree of freedom. We shall indeed concentrate in this section on the nonabelian subgroup $S U(N)$, and leave the abelian part to the next section.

In the presence of two Dirichlet p-branes, the calculation of the vector amplitude is modified only in two places: first of all, there is a zero mode correction in the integrand, namely:

$$
e^{-\frac{\tau y^{2}}{2 \pi^{2} \alpha^{\prime}}}
$$

where $y$ is the separation between the branes, and the rest of the notation is as before.

The second modification is physically much more important. The dimension $d$ we put before in the general expression (16) is now replaced by $d \equiv p+1$, the total worldvolume dimension of the brane, except that the string can vibrate in the full target spacetime, so that the exponent of Dedekind's function must be $D$ (that is 26 in the critical dimension).

In order to extract the field theory limit of this amplitude, we condider the simultaneous limit where $\alpha^{\prime}, y, \tau^{-1} \rightarrow 0$, keeping finite Maldacena's variable, $u \equiv y / \alpha^{\prime}$ as well as $\tilde{\tau} \equiv \alpha^{\prime} \tau$ and $\hat{\nu} \equiv \nu / \tau$.

It is worth remarking that the variables $\tilde{\tau}$ and $u$ are somewhat similar geometrically, owing to the fact that $y$ and $\tau^{-1}$ are respectively the world-sheet and space-time length of the cylinder.

To be specific, the on-shell amplitude now reads:

$$
A=\frac{g^{2}}{(4 \pi)^{d / 2}} \epsilon_{1} \cdot \epsilon_{2} \int \frac{d \bar{\tau}}{\bar{\tau}} \int_{0}^{1} d \hat{\nu} \bar{\tau}^{1-d / 2} e^{-u^{2} \bar{\tau}}\left[(D-2)(1-2 \bar{\nu})^{2}-8\right]
$$

From where we easily get:

$$
A=-\frac{g^{2}}{(4 \pi)^{d / 2}} \epsilon_{1} \cdot \epsilon_{2} \Gamma(1-d / 2)\left(u^{2}\right)^{d / 2-1}[(D-2)-4(D-2) B(2,2)-8]
$$

\footnotetext{
${ }^{5}$ Were the external momenta to have non-vanishing transversal components, Dirichlet Green functions ought to be employed.
} 
(where $B(u, v)$ is Euler's Beta function), which by standard dimensional regularization leads for $d=4$ to:

$$
A=-\frac{g^{2}}{(4 \pi)^{d / 2}} \epsilon_{1} \cdot \epsilon_{2} u^{2} \log \left(u^{2} / \Lambda^{2}\right)[(D-2)-4(D-2) B(2,2)-8]
$$

It has been already pointed out that Maldacena's variable $u$ plays naturally the rôle of the renormalization group scale (cf. [3]).

We notice finally, that, in contrast with (16), D-brane amplitudes can perfectly well be considered on shell, with external momenta parallel to the D-brane, and thus with only $d \equiv p+1$ non trivial components but, we insist, with oscillatory modes of the intermediate strings vibrating in all $D$ dimensions.

A further observation is that when computing the planar amplitude, the factor $\frac{6-7 d}{d-1}$ in eqn.(15) is now replaced by $\frac{D+6-8 d}{d-1}$. This in turn leads to the new beta function as given by:

$$
\beta(g)=\frac{1}{2} \frac{D+6-8 d}{d-1} C_{2}^{a d j}(G) \frac{g^{3}}{16 \pi^{2}}
$$

It is worth noticing that $\beta=0$ when $D=26$ and $d=4$, which corresponds to YangMills with $n_{s}=22$ scalar flavours in the adjoint of $S U(N)$ (99). Incidentaly, this theory is known not to be conformal at two loops (the beta function is instead positive). It would be exceedingly interesting to perform this calculation in a stringy context, to check whether the quantum field theory contribution to the two-loop beta function can be related to contributions coming from the region of the genus two string diagram in which closed string states are exchanged.

\section{Abelian groups in the infinite tension limit}

To start with we will reduce ourselves in this section to repeat our previous analysis for abelian gauge groups. What we should naturally expect as infinite tension field theory limit would be pure Maxwell theory without charged matter and therefore trivially vanishing 
beta function. This is what seems to indicate the field theory limit of tree level string amplitudes with abelian Chan-Paton factors, where for instance the three point amplitude vanishes as a consequence of cyclic invariance. When we try to check the validity of this picture going to one loop amplitudes we get some surprises that could be potentially interesting to understand the low energy field theory on the world volume of just one D-brane.

Coming back to the analysis of the two point amplitude nothing much changes in the Abelian case, except for the fact that the nonplanar diagram where the vertex operators are inserted in different boundaries does not vanish anymore. This means that the situation is in some sense, the opposite as in field theory: now we have two diagrams instead of only one. The non planar diagram in the full fleged string corresponds to the well known reggeon-pomeron vertex; a vertex where the open string without Chan-Paton factors becomes a closed string. In the limit $\tau=0$ the corresponding diagram is dominated by light closed string states that can mix with the photon giving rise to a stringy version of Higgs mechanism known as Cremmer Scherk [7] phenomena. In this paper we will not be interested in this limit ( that away from the critical dimension produces cuts violating unitarity ) but instead in the $\tau=\infty$ limit of the non planar diagram.

In order to capture the essential difference between abelian and non-abelian, let us also perform this computation in detail.

The first part of the calculation is already done; the planar diagram is exactly the same as before; with the only difference that the factor coming from the external traces is now $\left(\frac{1}{\sqrt{2}}\right)^{2}$ instead of $N / 2 \delta_{a b}{ }^{6}$

The nonplanar diagram can be similarly computed as:

$$
C_{N P}\left(p_{1}, p_{2}\right)=\frac{1}{2} \frac{g^{2} \mu^{4-d}}{(4 \pi)^{d / 2}}\left(2 \alpha^{\prime}\right)^{1-d / 2}
$$

\footnotetext{
${ }^{6}$ This is because if the $U(1)$ is considered as imbedded in $U(N)$, it is natural to keep the normalization of the generators in such a way that $\operatorname{tr} T^{2}=\frac{1}{2}$.
} 


$$
\begin{aligned}
& \int_{0}^{\infty} d \tau \int_{0}^{s} d \nu \tau^{-d / 2} e^{\frac{(26-d) \tau}{12}} \eta(i \tau / \pi)^{-(d-2)} \\
& {\left[-\left(\epsilon_{1} \cdot \epsilon_{2}\right) \partial_{\nu_{1}} \partial_{\nu_{2}} G_{P}\left(\nu_{1}, \nu_{2}\right)+\alpha^{\prime}\left(\epsilon_{1} \cdot p_{2}\right)\left(\epsilon_{2} \cdot p_{1}\right) \partial_{\nu_{1}} G_{N P}\left(\nu_{1}, \nu_{2}\right) \partial_{\nu_{2}} G_{N P}\left(\nu_{1}, \nu_{2}\right)\right]} \\
& e^{2 \alpha^{\prime} p_{1} \cdot p_{2} G_{N P}\left(\nu_{1}, \nu_{2}\right)}
\end{aligned}
$$

$G_{N P}\left(\nu_{1}, \nu_{2}\right)$ is the Green function computed between different boundaries (that is, $G_{N P} \equiv$ $\left.G\left(i \frac{\nu}{\pi}+\frac{1}{2} \mid i \frac{\tau}{\pi}\right)\right)$ ], namely:

$$
G_{N P}\left(\nu_{1}, \nu_{2}\right)=\log -2 \pi \frac{\theta_{2}\left(\frac{i}{\pi}\left(\nu_{2}-\nu_{1}\right) \mid \frac{i \tau}{\pi}\right)}{\theta_{1}^{\prime}\left(0 \mid \frac{i \tau}{\pi}\right)}-\frac{\left(\nu_{2}-\nu_{1}\right)^{2}}{\tau}
$$

The expansion of the Green function is:

$$
G_{N P} \sim-\frac{\nu^{2}}{\tau}+\nu+\log \left(1+e^{-2 \nu}\right)+e^{-2 \tau}\left[\frac{e^{-3 \nu}+e^{3 \nu}}{e^{\nu}+e^{-\nu}}+3\right]
$$

which yields for the product of the derivatives of the Green functions:

$$
\partial G_{N P} \partial G_{P} \sim(d-2)(1-2 \hat{\nu})^{2}+8 e^{-2 \tau}
$$

The last term cancels when added to the planar diagram, and the final result for the (divergent part of) the sum of the two contributions is:

$$
C_{N P}(s)=2 \frac{1}{2} \frac{g^{2}}{(4 \pi)^{2}} \frac{d-2}{d-1} \Gamma\left(\frac{4-d}{2}\right) s \epsilon_{1} . \epsilon_{2} B(1,1)
$$

leading tof :

$$
\beta=2 \frac{1}{48 \pi^{2}} g^{3}
$$

This is exactly the correct result for scalar QED with two $=(d-2)$ flavours in the fundamental representation of the abelian gauge group.!

It comes, however, as a surprise, because if we only had gauge particles in this limit, it being a free theory, we should expect $\beta=0$.

\footnotetext{
7 There is an extra factor of $i$ coming from the zero modes, cf. 10

${ }^{8}$ Actually the counting is very simple: the non surviving part of the factor $11 / 3$ is the non UV free, that is, $1 / 3$ and this appears twice, once for each string diagram
} 
The origin of the non trivial beta function goes back to the structure of string amplitudes where the Chan-Paton group labels appears as multiplicative factors. So for instance the planar diagram is , up to a numerical factor, the same for abelian and non abelian groups. The specific abelian contribution corresponding to the non planar diagram is not cancelling the planar amplitude since the zero mode part of $G$ and $G_{N P}$ are the same. What cancels between the planar and the non planar amplitudes is the piece coming from what we denote amplifying tachyon effect. Thus the field theory limit of string amplitudes with abelian Chan-Paton factors is not pure Maxwell but instead scalar QED, more precisely the scalar QED describing the diamagnetic part of the Yang Mills beta function.

If we repeat the previous analysis using a D-brane picture we get a beta function depending on the target space time dimension $D$ of the type:

$$
\beta(g)=\frac{D-2}{d-1} \frac{1}{16 \pi^{2}} g^{3}
$$

which for $d=4$ is that of QED with $(D-2)$ charged scalar particles. It is important to notice that the number of scalars contributing to the beta function is independent of the D-brane world volume dimension $d$. Notice also that contrary to what did happen for the non abelian case where we found $\beta=0$ for $D=26$ here we get $\beta=0$ for $D=2$ that curiously enough is the critical dimension for the $U(1)$ string model of [2].

The previous analysis of $U(1)$ amplitudes lead us naturally to the conclusion that the low energy field theory description of one D-3 brane is scalar QED with $(D-2)$ charged scalars. The physics underlying these extra scalars can be due to the dual interpretation of loop corrections in perturbation theory as tree level amplitudes where one of the states is a closed string state i.e part of the gravitational sector. It could be this interaction with gravity the one resposible for the non vanishing beta function and therefore for the unexpected new degrees of freedom. 


\section{Acknowledgments}

We are grateful to Ian Kogan for inspiring discussions. This work has been partially supported by the European Union TMR program FMRX-CT96-0012 Integrability, Nonperturbative Effects, and Symmetry in Quantum Field Theory and by the Spanish grant AEN96-1655. The work of E.A. has also been supported by the European Union TMR program ERBFMRX-CT96-0090 Beyond the Standard model and the Spanish grant AEN961664 .

\section{References}

[1] L.F. Abbott, The Background Field Method Beyond One Loop, Nucl. Phys. B185(1981), 189.

L.F. Abbott, M.T. Grisaru and R. K. Schaeffer, The Background Field Method and the S-Matrix, Nucl.Phys. B229,(1983),372.

[2] M. Ademollo et al., Dual String with U(1) color symmetry, Nucl.Phys.B111:77$110,1976$.

[3] E. Alvarez and C. Gómez, Noncritical confining strings and the renormalization group, hep-th 9902012; Nucl. Phys. B550 (1999),169.

[4] L. Alvarez Gaume, C. Gomez, G. Moore, C. Vafa, Strings in the Operator Formalism, Nucl.Phys.B303:455,1988.

[5] Zvi Bern, String-based Perturbative Methods for Gauge Theories, hep-th/9304249. (Presented at Theoretical Advanced Study Institute (TASI 92): From Black Holes and Strings to Particles, Boulder, CO, 3-28 Jun 1992). Published in Boulder TASI 92:0471-536 
[6] C.P. Burgess and T.R. Morris, Open and Unoriented Strings à la Polyakov, Nucl. Phys. B291(1987)256.

[7] E. Cremmer and J. Scherk, Spontaneous dynamical breaking of gauge symmetry in dual models, Nucl. Phys. B72 (1974) 117.

[8] P. Di Vecchia, L. Magnea, A. Lerda, R. Russo and R. Marotta, String Techniques for the Calculation of Renormalization Constants in Field Theory, Nucl. Phys. B469 (1996) 235; hep-th/9601143

[9] E.S. Fradkin and A. Tseytlin, Quantum properties of higher dimensional and dimensionally reduced supersymmetric theories, Nucl.Phys. B227 (1983) 252.

[10] M.B.Green.J.H.Schwarz and E. Witten, Superstring Theory (Cambridge University Press, 1987)

[11] D.J. Gross and F. Wilczek, Phys. Rev. Lett. 30 (1973)1343;

H.D. Politzer, Phys. Rev. Lett, 30 (1973)1346.

[12] R. Metsaev and A. Teytlin, On loop corrections to string theory effective actions, Nucl.Phys. B298 (1988) 109,

[13] J.A. Minahan, One-Loop Amplitudes on Orbifolds and the Renormalization of Coupling Constants, Nucl. Phys. B298 (1988),36.

[14] N.K. Nielsen, Asymptotic freedom as a spin effect, Am. J. Phys.49 (1981),1171. 